\title{
Closure of the saphenous opening after saphenofemoral junction disconnection: An anatomical barrier against postoperative neovascularization
}

\author{
Sherif Essam Tawfik, MD; Hesham Adel Alaa-Eldin, $M D$
}

\author{
Department of General Surgery, Faculty of Medicine, Ain Shams University, \\ Cairo, Egypt.
}

\section{Correspondence:}

Sherif Essam Tawfik, Department of Surgery, Faculty of Medicine, Ain Shams University, Tel.: +20101601731; fax: +20226177003

\begin{abstract}
Introduction: Neovascularisation at the sapheno-femoral junction (SFJ) ligation site in the groin may occur within one year after great saphenous vein $(G S V)$ surgery. Several anatomical and prosthetic barrier techniques have been proposed to prevent this evolution.

Aim: To test the hypothesis that the construction of an anatomical barrier by simple closure of the saphenous opening might decrease the incidence and extent of postoperative neovascularisation at the SFJ.

Patients and methods: From October 2006 to October 2008, we included 112 patients with 130 limbs presenting with primary varicose veins. The test (group A) patients, had the edges of the saphenous opening closed after SFJ disconnection. Follow up was done by duplex scan to detect neovascular serpentine veins at the groin.

Results: Our patients mean age was 30.2 years. According to preoperative CEAP classification we had 76 limbs classified as C2, and 54 limbs classified as C3. Both groups were initially matched regarding the venous disability score (VDS). There was a significant difference $(p=0.025)$ between the 2 groups at 1 year duplex follow up with 2 patients in the test group (group A) with groin neovascularization versus 12 patients in the control group (group B).

Conclusion: This study suggests a potential benefit of closure of the saphenous opening after SFJ ligation in the groin, a technique applied to contain postoperative neovascularisation at the ligated GSV stump.

Keywords: Neovascularization; Saphenofemoral.
\end{abstract}

\section{Introduction:}

Recurrent varices after surgery (REVAS) is a common, complex, and costly problem for both the patient and the surgeon who treats them. ${ }^{1}$ Some causes of recurrence are obvious: insufficient understanding of venous anatomy and hemodynamics, inadequate preoperative assessment, and incorrect or insufficient surgery. However, recurrence at the SFJ cannot always be explained by technical inadequacy of the original surgical intervention. Its development has also been attributed to neovascularization in the granulation tissue around the ligated stump. ${ }^{2}$ Neovascularization at the level of the ligated sapheno-femoral junction (SFJ) is now recognized as one of the important pathophysiological mechanisms leading to recurrence of varicose veins. ${ }^{3-6}$

Neovascularization is defined as new blood vessel formation (angiogenesis) occurring in the surrounding tissue which may be induced by diffusible chemical factors (angiogenic factors). In the particular context of varicose recurrence after great saphenous vein (GSV) surgery, the term neovascularization describes a phenomenon of formation of new venous 
channels between the saphenous stump on the common femoral vein (CFV) and the residual GSV or its tributaries. ${ }^{7}$

Duplex-based prospective studies revealed some degree of neovascularization represented by typical serpentine tributaries arising from the ligated SFJ in up to $14 \%$ of operated limbs at one year after flush saphenofemoral or saphenopopliteal junction ligation. ${ }^{8}$ To contain such neovascularisation and hence prevent recurrence from the groin the use of a prosthetic or anatomical barrier to cover the ligated saphenous stump has been proposed.9-19 However, implantation of foreign material may lead to postoperative complications. Therefore systematic use of a prosthetic patch in the groin after SFJ ligation remains a questionable issue. Construction of a simple anatomical barrier without implantation of foreign material might offer a valuable alternative. The easiest approach to construct such anatomical barrier consists of closure of the saphenous opening once the SFJ has been ligated. The saphenous opening, in the groin is the deep fascial defect traversed by the GSV at its termination before it joins the common femoral vein (CFV).

For this study we hypothesised that the construction of an anatomical barrier by simple closure of the saphenous opening might decrease the incidence and extent of postoperative neovascularization at the SFJ. For the sake of critical evaluation of this anatomical barrier technique, we conducted this comparative study to compare a group of patients with the anatomical barrier technique to another cohort of patients without the anatomical barrier technique after SFJ disconnection.

\section{Patients and methods:}

Over a period of 24 months from October 2006 to October 2008, we included 112 patients with 130 limbs presenting with primary varicose veins to Ain Shams University Hospitals, and Nasr City Health Insurance Hospital. They were assigned in consecutive order into group A (closure of saphenous opening) or group B (no closure of saphenous opening). In case of bilaterality, the 2 limbs were assigned to 2 different treatment groups. We included patients with primary varicose veins due to incompetent SFJ, diagnosed on clinical grounds and documented by venous duplex scan.

Our inclusion criteria based on CEAP classification included:

1. Patients without extensive clinical symptoms consistent with $\mathrm{C} 2$ and $\mathrm{C} 3$ categories (varicose veins with or without edema but without skin changes).

2. Etiological classification Ep (primary varicose veins).

3. Anatomical classification As (affecting the superficial system)

4. Pathophysiological classification Pr (reflux).

Patients were clinically evaluated preoperatively with the venous disability score (VDS) Table(1) together with duplex mapping with special emphasis on the SFJ to point out any anatomical variations at the SFJ (e.g. double saphenous). Patients with bilateral varicose veins were operated upon with 3 months interval between both legs.

Table (1):Venous disability score (VDS).

\begin{tabular}{|c|l|}
\hline 0 & Asymptomatic \\
\hline 1 & Symptomatic but able to carry out usual activities* without compressive therapy. \\
\hline 2 & Can carry out usual activities* only with compression and/or limb elevation. \\
\hline 3 & Unable to carry out usual activities* even with compression and/or limb elevation. \\
\hline
\end{tabular}

*Usual activities = patient's activities before onset of disability from venous disease. 
Surgical procedure was standardized as transverse groin incision at site of SFJ which was marked preoperatively by duplex scan. Identification of GSV at its termination into the common femoral vein, and ligation of tributaries ending at the SFJ. Over sewing of the SFJ stump by a nonabsorbable Prolene taper point 3-0 monofilament suture.

For group A patients, meticulous identification and careful closure of the edges of the saphenous opening by 2-3 interrupted nonabsorbable Prolene taper point 3-0 monofilament suture was the differentiating step Figure(1). For both groups, surgery proceeded afterwards by closure of the membranous layer of the subcutaneous tissue before skin closure. This was followed by stab avulsions for the leg varicosities, and finally GSV stripping to the knee level.

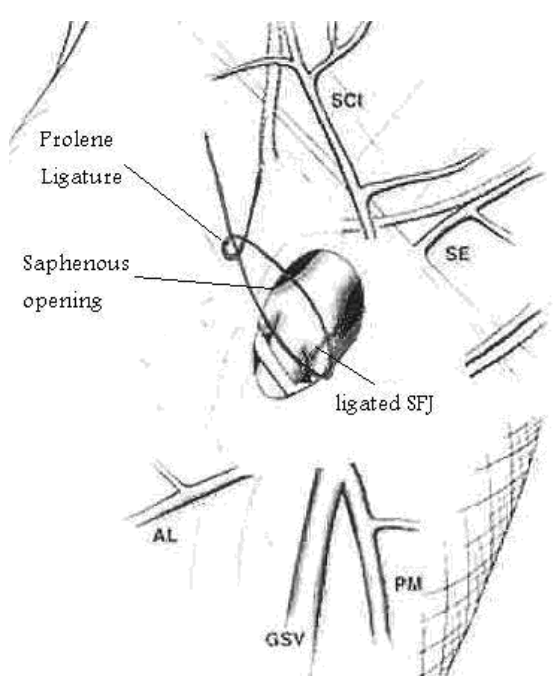

(B)

Figure (1): A) SFJ before disconnection traversing the saphenous opening, B) Prolene stitch going through the edges of the saphenous opening after SFJ disconnection.

Postoperatively the patient used elastic stockings for 3 weeks. All patients were given subcutaneous Clexane (Enoxaparine sodium) $20 \mathrm{mg}$ once daily starting from the night before surgery and for 1 postoperative day. Follow up was scheduled at 2 months and 12 months postoperatively for clinical assessment by venous disability score (VDS), and duplex evaluation for the sake of identification of neovascular serpentine tributaries at the groin together with quantification of these tributaries according to its size into G0 (no serpentine tributaries), G1 (tiny serpentine veins $<2 \mathrm{~mm}$ ), G2 (serpentine veins $>2 \mathrm{~mm}$ diameter). Duplex was done by two dedicated operators following the above mentioned simple quantification protocol. Complications in the early postoperative period or during follow up were documented.

\section{Results:}

A total of 112 patients with 130 limbs were included. There were 94 patients with unilateral disease and 18 patients with bilateral lower limb affection. We had 74 males and 38 females. Their mean age was 30.2 years. According to preoperative CEAP classification we had 76 limbs classified as C2, and 54 limbs classified as C3. On initial evaluation, the venous disability score (VDS) was 1 for 30 affected limbs, and VDS of 2 for 65 of the affected limbs, and VDS of 3 for 35 of the affected limbs. The characteristics of patients and limbs are represented in Table(2). 
Table (2): Characteristics of patients and limbs.

\begin{tabular}{|l|c|}
\hline Patients & $\mathrm{n}=112$ \\
\hline Limbs & $\mathrm{n}=130$ \\
\hline Age & $30.2(+/-8.1)$ \\
\hline Women & $38(34 \%)$ \\
\hline Bilateral disease & $18(16 \%)$ \\
\hline C2 classification & $76(58.5 \%)$ \\
\hline C3 classification & $54(41.5 \%)$ \\
\hline VDS $=1$ & 30 \\
\hline VDS $=2$ & 65 \\
\hline VDS $=3$ & 35 \\
\hline
\end{tabular}

The VDS score shown in Table(3), shows that both groups were matching regarding the VDS at initial presentation, but there was a significant difference at 1 year follow up $(\mathrm{p}=0.024)$ with group A patients having a lower venous disability score (VDS) than group B patients. At 1 year follow up, we had 7 patients lost for follow up, one of them with bilateral lower limb affection, 2 patients with unilateral lower limb affection from group A, and 4 patients with unilateral lower limb affection from group B. This left us with 62 limbs in group A, and 60 limbs in group B.

\section{Table (3): VDS follow up at 1 year.}

\begin{tabular}{|c|c|c|c|c|c|c|c|c|c|}
\hline & \multicolumn{4}{|c|}{ Group A } & \multicolumn{4}{|c|}{ Group B } & $P$ value \\
\hline VDS & 0 & 1 & 2 & 3 & 0 & 1 & 2 & 3 & \\
\hline initial & & 13 & 36 & 16 & & 17 & 29 & 19 & 0.46 \\
\hline 12 months & 53 & 9 & & & 41 & 19 & & & 0.024 \\
\hline
\end{tabular}

Regarding duplex follow up for groin neovascularization at 2 months there was no detectable serpentine neovascular venous channels detected in both groups. However, there was a highly significant difference $(p=0.025)$ between the 2 groups at 1 year duplex follow up for groin neovascularization. There were 2 limbs with grade 1 neovascularization in group A, while there were 4 limbs with grade 1 and 8 limbs with grade 2 neovascularization in group $\mathrm{B}$. This data is represented in Table(4).

Table (4): Groin neovascularization at 2 and 12 months follow up.

\begin{tabular}{|l|c|c|c|c|c|c|c|}
\hline & \multicolumn{3}{|c|}{ Group A } & \multicolumn{3}{c|}{ Group B } & P value \\
\hline Neovasc. & 0 & 1 & 2 & 0 & 1 & 2 & \\
\hline 2 months & 65 & & & 65 & & & \\
\hline 12 months & 60 & 2 & & 48 & 4 & 8 & 0.025 \\
\hline
\end{tabular}


Regarding the 18 patients who had bilateral disease, where each limb was allocated to a different treatment group, we did a subgroup analysis for them to see the difference in duplex detected neovascularization at 1 year. One of the 18 patients was lost to follow up.
Table(5) shows that there was a significant difference $(p=0.033)$ regarding groin neovascularization at 1 year, between the limbs allocated to the two treatment groups, in this subgroup of patients with bilateral disease.

Table (5): Groin neovascularization for the bilateral disease subgroup.

\begin{tabular}{|l|c|c|c|c|c|c|c|}
\hline & \multicolumn{3}{|c|}{ Group A } & \multicolumn{3}{c|}{ Group B } & P value \\
\hline Neovasc. & 0 & 1 & 2 & 0 & 1 & 2 & \\
\hline 2 months & 17 & & & 13 & 4 & & 0.033 \\
\hline
\end{tabular}

In the early post operative period there were few minor complications in the form of 6 groin wound infection; 2 of them in group A, and 4 in group B. No saphenous nerve injury reported. Bruising along the track of GSV stripping was not considered as a complication as it invariably disappeared at a maximum period of 3 weeks.

\section{Discussion:}

This study aimed at evaluation of closure of saphenous opening after SFJ ligation as an anatomical barrier to reduce the incidence of groin neovascularization. The study revealed an incidence of postoperative neovascularisation at the SFJ of $3.2 \%$ after one year compared to $20 \%$ in the no closure group. Very few studies have addressed the potential usefulness of anatomical barriers to contain postoperative neovascularization and lower the postoperative recurrence rate. The majority of these studies date from before the era of duplex scanning, which makes comparison difficult. Glass ${ }^{10,12}$ studied the effects of closure of the cribriform fascia on the incidence of clinical recurrence after SFJ ligation and multiple ligations of the GSV (without stripping). At least 4 years after the initial operation, recurrence from the previous SFJ ligation site was confirmed at surgical reexploration in $25 \%$ of 141 limbs initially operated without closure of the cribriform fascia and in $3 \%$ of 127 limbs operated with closure of this fascia. Thomson ${ }^{11}$ suggested closing the saphenous opening by raising a flap of fascia from the fascial tunnel of the GSV and folding it cephalad to be stitched in place around the opening. Reviewing the results of 137 such consecutive operations, he found only two recurrences (however no postoperative time interval was mentioned). Many years before, the construction of an alternative anatomical barrier designed to prevent recurrence after an efficiently performed high ligation had been suggested. ${ }^{9}$ The technique was slightly more complicated and required a more extensive dissection in the groin. It consisted of suturing a flap of pectineus fascia over the common femoral vein and ligated GSV stump to the femoral sheath and margins of the saphenous opening. In 1978, Sheppard had reported 'encouraging results' with this type of intervention. ${ }^{9}$

Concerning the use of a silicone implant and closure of the cribriform fascia, there had been promising results with this technique. 16,18 Unfortunately this technique resulted in some short- and long-term complications potentially related to the foreign material used: not only some early and late postoperative infections and lymphatic problems, but also some cases of venous thromboembolism and of abnormal scar tissue reaction causing a symptomatic stenosis of the common femoral vein. ${ }^{20}$

In our study, the fact that follow-up period was for one year, is considered short in terms of recurrence as clinically visible or palpable recurrent varicose veins usually become apparent only after three to five years. However, early ultrasound scanning after one year permits early detection of 'duplex-recurrence', which appears as different degrees of neovascular veins at the site of the previous SFJ 
ligation. ${ }^{3,8,21}$ Duplex-based prospective studies revealed some degree of neovascularization represented by typical serpentine tributaries arising from the ligated SFJ in up to $14 \%$ of operated limbs at one year after flush saphenofemoral or saphenopopliteal junction ligation. ${ }^{8}$ It has been shown that these early postoperative duplex findings help to predict which patients will develop recurrence and potentially require re-operation (or alternative treatment) in the long term. ${ }^{21,22}$

To avoid postoperative neovascularisation, alternative techniques with the aim of obliterating the GSV (endovenous procedures with radiofrequency or laser energy) without groin dissection have shown promising results. ${ }^{23,24,25}$ After radiofrequency obliteration of the GSV duplex ultrasound scans confirmed the absence of neovascular veins in the groin. ${ }^{26,27}$ The obvious advantage of these techniques consists in the fact that there is no groin incision and hence no surgical trauma, which is a potential trigger for neovascularization. In addition, in the majority of cases the superficial epigastric vein remains patent after endovenous obliteration of the GSV, with the advantage of not disturbing drainage of the abdominal wall, in this way avoiding an additional trigger for neovascularization. ${ }^{5}$ However, technical equipment for endovenous procedures is not yet available in all surgical centres, as these devices and the catheters used are quite expensive. In such patients, well performed surgery will still offer a valuable alternative. Closing the saphenous opening after SFJ ligation in these cases might reduce postoperative neovascularization in the groin and hence recurrence of varicose veins.

The use of VDS score in our study was intended to measure the patients' symptomatic response after surgery in each group independently. However, the fact that there was a significant difference at 1 year follow up $(\mathrm{p}=0.024)$ with group A patients having a lower venous disability score (VDS) than group B patients, cannot be explained by the sole duplex finding of groin neovascularization. Thus a larger study with a larger sample, clinical severity scoring, and "quality of life" assessment, is probably needed to evaluate the effect of this modified surgical technique on the symptomatic outcome and quality of life.

In conclusion, this study suggests a potential benefit of closure of the saphenous opening after SFJ ligation in the groin, a technique applied to contain postoperative neovascularization at the ligated GSV stump. This simple technical is an adjunctive measure to reduce the incidence of recurrence in those patients who still undergo 'classic' varicose veins operations.

\section{References:}

1- Michel Perrin: Classification and treatment of recurrent varicose veins. In: The Vein Book. John J Bergan, (Editor); Elsevier (Publisher); 2007; p. 299-307.

2- De Maeseneer MG: The role of postoperative neovascularization in recurrence of varicose veins: From historical background to today's evidence. Acta Chir Belg 2004; 104: 283-289.

3- Jones L, Braithwaite BD, Selwyn D, Cooke S, Earnshaw JJ: Neovascularization is the principal cause of varicose vein recurrence: Results of a randomised trial of stripping the long saphenous vein. Eur J Vasc Endovasc Surg 1996; 12: 442-445.

4- De Maeseneer MG, Tielliu IF, Van Schil PE, De Hert SG, Eyskens EJ: Clinical relevance of neovascularization on duplex ultrasound in the long term follow up after varicose vein operation. Phlebology 1999; 14: 118-122.

5- Fischer R, Chandler JG, De Maeseneer MG, Frings N, Lefebvre-Vilardebo M, Earnshaw $\mathrm{JJ}$, et al: The unresolved problem of recurrent saphenofemoral reflux. J Am Coll Surg 2002; 195: 80-94.

6- Van Rij AM, Jones GT, Hill GB, Hons BS, Jiang P: Neovascularization and recurrent varicose veins: More histologic and ultrasound evidence. J Vasc Surg 2004; 40: 296-302.

7- De Maeseneer MG: Neovascularization: An adverse response to proper groin dissection. In: The Vein Book. John J Bergan, (Editor); Elsevier (Publisher); 2007; p.239-245.

8- De Maeseneer MG, Ongena KP, Van den Brande F, Van Schil PE, De Hert SG, Eyskens EJ: Duplex ultrasound assessment 
of neovascularization after sapheno-femoral or sapheno-popliteal junction ligation. Phlebology 1997; 12: 64-68.

9- Sheppard M: A procedure for the prevention of recurrent saphenofemoral incompetence. Aust NZ J Surg 1978; 48: 322-326.

10-Glass GM: Prevention of recurrent saphenofemoral incompetence after surgery for varicose veins. Br J Surg 1989; 76:1210.

11-Thomson H: Saphenous vein stripping and quality of outcome. Br J Surg 1997; 84: 424-425.

12-Glass GM: Prevention of sapheno-femoral and sapheno-popliteal recurrence of varicose veins by forming a partition to contain neovascularization. Phlebology 1998; 13: 3-9.

13-Earnshaw JJ, Davies B, Harradine K, Heather BP: Preliminary results of PTFE patch saphenoplasty to prevent neovascularization leading to recurrent varicose veins. Phlebology 1998; 13:1013.

14-Gibbs PJ, Foy DM, Darke SG: Reoperation for recurrent saphenofemoral incompetence: A prospective randomised trial using a reflected flap of pectineus fascia. Eur $J$ Vasc Endovasc Surg 1999; 18: 494-498.

15-Bhatti TS, Whitman B, Harradine K, Cooke SG, Heather BP, Earnshaw JJ: Causes of re-recurrence after polytetrafluoroethylene patch saphenoplasty for recurrent varicose veins. Br J Surg 2000; 87: 1356-1360.

16-De Maeseneer MG, Giuliani DR, Van Schil PE, De Hert SG: Can interposition of a silicone implant after sapheno-femoral ligation prevent recurrent varicose veins? Eur J Vasc Endovasc Surg 2002; 24: 445449.

17-Creton D: Surgery for recurrent saphenofemoral incompetence using expanded polytetrafluoroethylene patch interposition in front of the femoral vein: Long term outcome in 119 extremities. Phlebology. 2002; 16: 93-97.

18-De Maeseneer MG, Vandenbroeck CP, Van Schil PE: Silicone patch saphenoplasty to prevent repeat recurrence after surgery to treat recurrent saphenofemoral incompetence: Long-term follow-up study. J Vasc Surg 2004; 40: 98-105.
19-Van Rij AM, Hill G, Christie R, Jones GT, Amer M, Thomson IAT, et al: Prevention of recurrence after saphenofemoral ligation: A randomised trial of mechanical inhibition of neovascularisation: Early results. Int Angiol 2006; 25(Suppl.1): 150.

20-De Maeseneer MG, Vandenbroeck CP, Lauwers PR, Hendriks JM, De Hert SG, Van Schil PE: Early and late complications of silicone patch saphenoplasty at the saphenofemoral junction. J Vasc Surg 2006; 44: 1285-1290.

21-Van Rij AM, Jiang P, Solomon C, Christie RA, Hill GB: Recurrence after varicose vein surgery: A prospective long-term clinical study with duplex ultrasound scanning and air plethysmography. $J$ Vasc Surg 2003; 38: 935-943.

22-De Maeseneer MG, Vandenbroeck CP, Hendriks JM, Lauwers PR, Van Schil PE: Accuracy of duplex evaluation one year after varicose vein surgery to predict recurrence at the sapheno-femoral junction after five years. Eur J Vasc Endovasc Surg 2005; 29: 308-312.

23-Min RJ, Khilnani N, Zimmet SE: Endovenous laser treatment of saphenous vein reflux: Long-term results. $J$ Vasc Interv Radiol 2003; 14: 991-996.

24-Merchant RF, Pichot O: Closure Study Group: Long-term outcomes of endovenous radiofrequency obliteration of saphenous reflux as a treatment for superficial venous insufficiency. J Vasc Surg 2005; 42: 502509.

25-Agus GB, Mancini SIEWG: The first 1000 cases of Italian Endovenous-laser Working Group (IEWG): Ratioanale, and long-term outcomes for the 1999-2003 period. Int Angiol 2006; 25: 209-215.

26-Pichot O, Kabnick LS, Creton D, Merchant RF, Schuller-Petrovic S, Chandler JG: Duplex ultrasound scan findings two years after great saphenous vein radiofrequency endovenous obliteration. J Vasc Surg 2004; 39: 189-195.

27-Kianifard B, Holdstock JM, Whiteley MS: Radiofrequency ablation (VNUS closure) does not cause neovascularization at the groin at one year: Results of a case controlled study. Surgeon 2006; 4: 71-74. 
28-Rautio T, Ohinmaa A, Perälä J, Ohtonen P, Heikkinen T, Wiik H, et al: Endovenous obliteration versus conventional stripping operation in the treatment of primary varicose veins: A randomized controlled trial with comparison of the costs. J Vasc Surg 2002; 35: 958-965. 